doi:10.1016/j.ecoenv.2005.07.023

Copyright (c) 2005 Elsevier Inc. All rights reserved.

\title{
Occurrence of phthalate esters in the environment of the Netherlands
}

\section{Willie J.G.M. Peijnenburg* and Jaap Struijs}

Laboratory for Ecological Risk Assessment, National Institute of Public Health and the Environment, P.O. Box 1, 3720 BA Bilthoven, The Netherlands

* Corresponding author. Fax: +31302744413.

\section{Abstract}

Overviews of levels of $n$-dibutylphthalate (DBP) and di(2-ethylhexyl)phthalate (DEHP) found in freshwater, marine water, sediment, and fish in the Netherlands are given. Sampling spanned a 9-month period (all seasons except winter) and allowed assessing whether phthalate levels are season dependent. Results obtained are compared to data reported for other Western European countries and a fugacity-based modeling approach is used to assess whether there is equilibrium among the various compartments. Highest levels of dissolved DBP and DEHP were found in freshwater samples, whereas these compounds were usually below the limit of detection (LOD) in marine water and sediment. Median levels were log-normally distributed; statistical analysis showed that sampling season is not a relevant determinant parameter. Similar results were obtained for the freshwater sediment compartment, with DEHP levels exceeding concentrations of DBP. DBP levels in fish were often below the LOD. Nevertheless, mean values around $1.8 \mu \mathrm{g} \mathrm{kg}^{-1}$ wet fish were found for both DEHP and DBP.

Fugacity calculations revealed that especially for DEHP there is no equilibrium among the compartments. DEHP emissions are directed to water, whereas the calculations reveal that sediments provide a sink for DEHP and there is net transport to air. Although it has been suggested that water is the primary compartment for DBP, fugacity plots suggest that air is the compartment to which emissions are directed dominantly. The data reported are in line with values found in Western Europe.

\section{Introduction}

Phthalate esters are industrial chemicals that are widely used as plasticizers in polyvinyl chloride resins (ECETOC, 1988). In the EU approximately 1,000,000 tons per year are produced $(4,300,000$ tons per year worldwide) which has been at a constant level for 20 years. Of the 18 commercial phthalate esters, the share of di(2-ethylhexyl)phthalate (DEHP) is about $50 \%$. Because of their properties, high production volumes, and widespread use, concentrations in various environmental compartments are measurable and expected to reflect a constant and diffuse release into the environment. DEHP and $n$-dibutylphthalate (DBP) are the phthalate esters that are most commonly reported as being detected in environmental samples. The consumption of phthalates can be estimated on a per capita basis, allowing calculation of emission data for a certain region with a known population density.

It was the purpose of this study to document levels of phthalate esters typically present in the Dutch aquatic environment. In addition, whether there is physical 
equilibrium with regard to phthalate concentrations detected was examined. The latter aspect is discussed by means of fugacity calculations based upon the physicochemical properties of the phthalates investigated. The discussion is restricted to the phthalates DBP and DEHP. Not only are these compounds the most abundant phthalate isomers typically found in the aquatic environment, but DBP and DEHP are also the phthalates for which the database was most complete with regard to the assessment of the various phthalates in the different environmental compartments. Most of the data on DEHP and DBP levels in the water column presented here were previously presented in a Dutch report by Vethaak et al. (2002). This database is supplemented with newly measured data on phthalate levels in fish, freshwater sediment, soil, and air.

The assessment of whether there is chemical equilibrium with regard to DEHP and DBP in the environmental compartments sampled is based upon the physicochemical properties of the compounds discussed here. For this purpose, a short overview of the physicochemical properties of DEHP and DBP is presented. Finally, the concentrations found in the Dutch aquatic environment are compared to phthalate levels typically found in other Western European countries.

At ambient temperature, DEHP and DBP are nonvolatile liquids with high boiling points. The air-water partition coefficient of both chemicals is well below 0.001 at $20^{\circ} \mathrm{C}$. Data in Table 1 were retrieved from a comprehensive review of physicochemical properties, environmental partitioning, bioaccumulation, and (a)biotic transformation processes by Staples et al. (1997). In Table 1, uncertainty due to empirical inaccuracy is expressed as $k$ (dispersion factor or uncertainty factor), assuming that the distribution of a physicochemical property is log-normal. The octanol-water partition coefficient $\left(K_{\text {ow }}\right)$ is related to the water solubility through a regression equation (Lyman et al., 1990). Literature values of $K_{\text {ow }}$ were slightly adjusted to link the tabulated values for water solubility and log$K_{\text {ow }}$ to each other, using these generally applicable and reliable regression equations.

Table 1.

Physicochemical properties of DEHP and DBP as reported by Staples et al. (1997)

\begin{tabular}{|l|l|l|l|l|l|}
\hline Property & Unit & DEHP & \multicolumn{2}{l|}{ DBP } \\
\hline & & Median & $k$ & Median & $k$ \\
\hline Molecular weight & $\mathrm{g} \mathrm{mol}^{-1}$ & 390.54 & - & 278.34 & - \\
\hline Vapor pressure, $t=25^{\circ} \mathrm{C}$ & $\mathrm{Pa}$ & $1.33 \times 10^{-5}$ & 2 & $3.6 \times 10^{-3}$ & 2 \\
\hline Solubility, $t=25^{\circ} \mathrm{C}$ & $\mathrm{mg} \mathrm{L}$ & $0.003^{\mathrm{a}}$ & 2 & $11.2^{\mathrm{a}}$ & 2 \\
\hline$K_{\text {ow }}$ & - & $10^{7.5}$ & 4.5 & $10^{4.5}$ & 3 \\
\hline Melting point & ${ }^{\circ} \mathrm{C}$ & -47 & - & -35 & - \\
\hline Partition coefficient air-water $(=H / R T)$ & - & $7.4 \times 10^{-4}$ & 2.7 & $3.8 \times 10^{-5}$ & 2.7 \\
\hline Partition coefficient suspended particles-water $\left(K_{p, \text { susp. }}\right)$ & $\mathrm{L} \mathrm{kg}^{-1}$ & $2.67 \times 10^{4}$ & 4.5 & $1.54 \times 10^{3}$ & 10 \\
\hline Partition coefficient sediment-water $\left(K_{\mathrm{p}, \text { sed. }}\right)$ & $\mathrm{L} \mathrm{kg}^{-1}$ & $2.78 \times 10^{4}$ & 7 & $4.53 \times 10^{2}$ & 27 \\
\hline Partition coefficient soil-water $\left(K_{\mathrm{p}, \text { soil }}\right)$ & $\mathrm{L} \mathrm{kg}^{-1}$ & $2.78 \times 10^{4}$ & 7 & $4.53 \times 10^{2}$ & 27 \\
\hline
\end{tabular}

The dispersion or uncertainty factor $k$ reflects the uncertainty accompanied with each property. $k$ expresses the uncertainty in the given median value as a ratio. Hence the uncertainty in the median is equal to median/ $k$-median* $k$.

${ }^{a}$ Correlated to uncertainty in $K_{\text {ow }}$ (corr. coeff. $=-0.96$ on log-scale). 
The atmospheric mixture of mainly nitrogen and oxygen, with an average molecular weight of $28.8 \mathrm{~g} \mathrm{~mol}^{-1}$, has a lower density than atmospheric DEHP and DBP. The molecular weight of DEHP is more than that of an order of magnitude higher than that of the air mixture, with the implication that the rarefacation pattern (i.e., mixing pattern) considerably deviates from that of major components in ambient air. In undisturbed atmosphere, the concentration of DEHP at $1000 \mathrm{~m}$ height is only $23 \%$ of the concentration at sea level (DBP $37 \%$ ), while the nitrogen-oxygen mixture still has $90 \%$ of its sea level pressure. Wind is a counteracting factor but during quiet weather a considerably higher concentration is accumulated on the ground. This may increase the variability of measured air concentrations sampled under varying weather conditions.

The partition coefficient $\left(K_{\mathrm{p}}\right)$ is defined as the ratio of the concentrations of the chemical in the solid and in the aqueous phases. $K_{\mathrm{p}}$ values related to solids-water partitioning are log-normally distributed. The median value used in this study and the uncertainty factor $(k)$ are based on data gathered by Staples et al. (1997). These data pertain to the organic carbon normalized partition coefficients, which are multiplied by the organic carbon content of the solids, $f_{\text {oc }}$, to obtain $K_{\mathrm{p}}$. Table 1 pertains to $K_{\mathrm{p}}$ values with $f_{\text {oc }}$ equal to 0.1 . The latter value corresponds to the 50th percentile of all EU soils (EURAS, Gent, Belgium; pers. comm.).

\section{Materials and methods-explanation of the sampling campaign}

\subsection{General}

Sites were selected to encompass both noncontaminated areas expected to display levels of phthalate esters at ambient concentrations and areas expected to be impacted by potential sources of contamination such as domestic and industrial sewage plants and rivers impacted by emission sources outside of the Netherlands. The locations of the sampling sites are given in Fig. 1 and Fig. 2. During sampling and further processing of the samples, precautions were taken to avoid contamination. This is especially important since phthalates are ubiquitous chemicals in indoor environments. In view of their use as plasticizers, it was ensure that no plastic equipment was used during sampling and that processing and all glassware used was new and precleaned. All equipment used was cleaned between samples. Detergents were not used during sample processing and all equipment was preconditioned and checked for blank levels of phthalates before sampling. Reagents used were of the highest analytical purity (analytical grade). Calibration was done using a six point calibration curve for each of the monitored phthalates, including $10,50,100,250,500$, and $1000 \mathrm{pg}^{-1}$. Each calibration sample contained $500 \mathrm{pg}^{-1} \mathrm{~L}^{-1}$ internal standard (d4-DEHP). The calibration curves had correlation coefficients greater than 0.995. Data are blank corrected. Instrument blanks were controlled by a $1-\mu \mathrm{L}$ injection of pure cyclohexane solvent. Phthalates should not be detected in concentrations larger than $5 \mathrm{pg} \mathrm{\mu L}^{-1}$ in the blank (peak area $<50 \%$ of peak area of a $10-\mathrm{ppb}$ calibration standard). The limit of detection (LOD) is determined by the method blank. The limit of quantitation (LOQ) was set at $2 \times$ the blank value. One of the parameters which determine the LOQ is the analytical (instrumental) detection limit. This was determined by the GC conditions (injection) and the sensitivity of the mass spectrometer. The sensitivity was measured by the injection of a $10-\mathrm{pg} \mu \mathrm{L}^{-1}$ solution, and the signal-to-noise ratio should be greater than 6 . The recovery was tested by spiking an olive oil sample at the 500-ppb level with DBP and DEHP. Olive oil was selected as a nonpolar solvent in which DBP and DEHP are easily dissolved. No phthalates were detected in the olive oil at concentrations above $50 \mathrm{ppb}$. This oil was therefore considered the "blank". d4-DEHP (internal standard) was added to the dichloromethane solution. The sample was analyzed 
in threefold. The concentrations were determined using the GC-MS calibration curves (ion 149 was monitored) and compared to the spiked concentration. In general, good recoveries ( $>80 \%$ ) were obtained. The linearity was tested by spiking an olive oil sample at eight levels $(0,100,250,500,1000,2500,5000$, and 7500-ng g ${ }^{-1}$ fat). The olive oil was tested and no phthalates were detected at concentrations above $50 \mathrm{ppb}$. The correlation coefficients were greater than 0.99 for all phthalates. This correlation shows that the method can be used in the concentration range $100-7500 \mathrm{ppb}$.

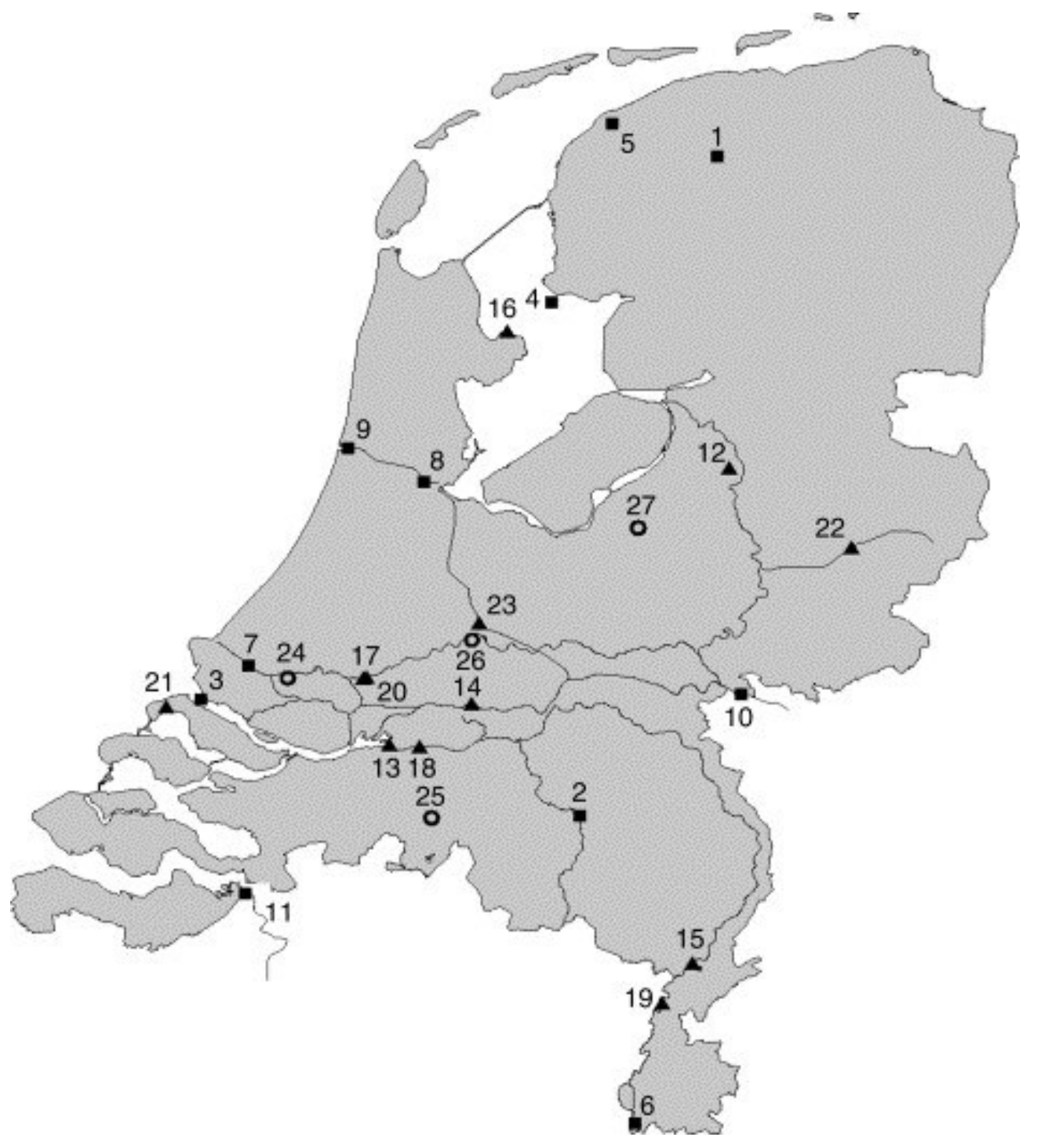

Fig. 1. Overview of sampling locations in the Netherlands for fresh surface water (black triangles: water only; black squares: both water and suspended matter). The sites coded " $\mathrm{O}$ " represent the locations where air samples were taken. 


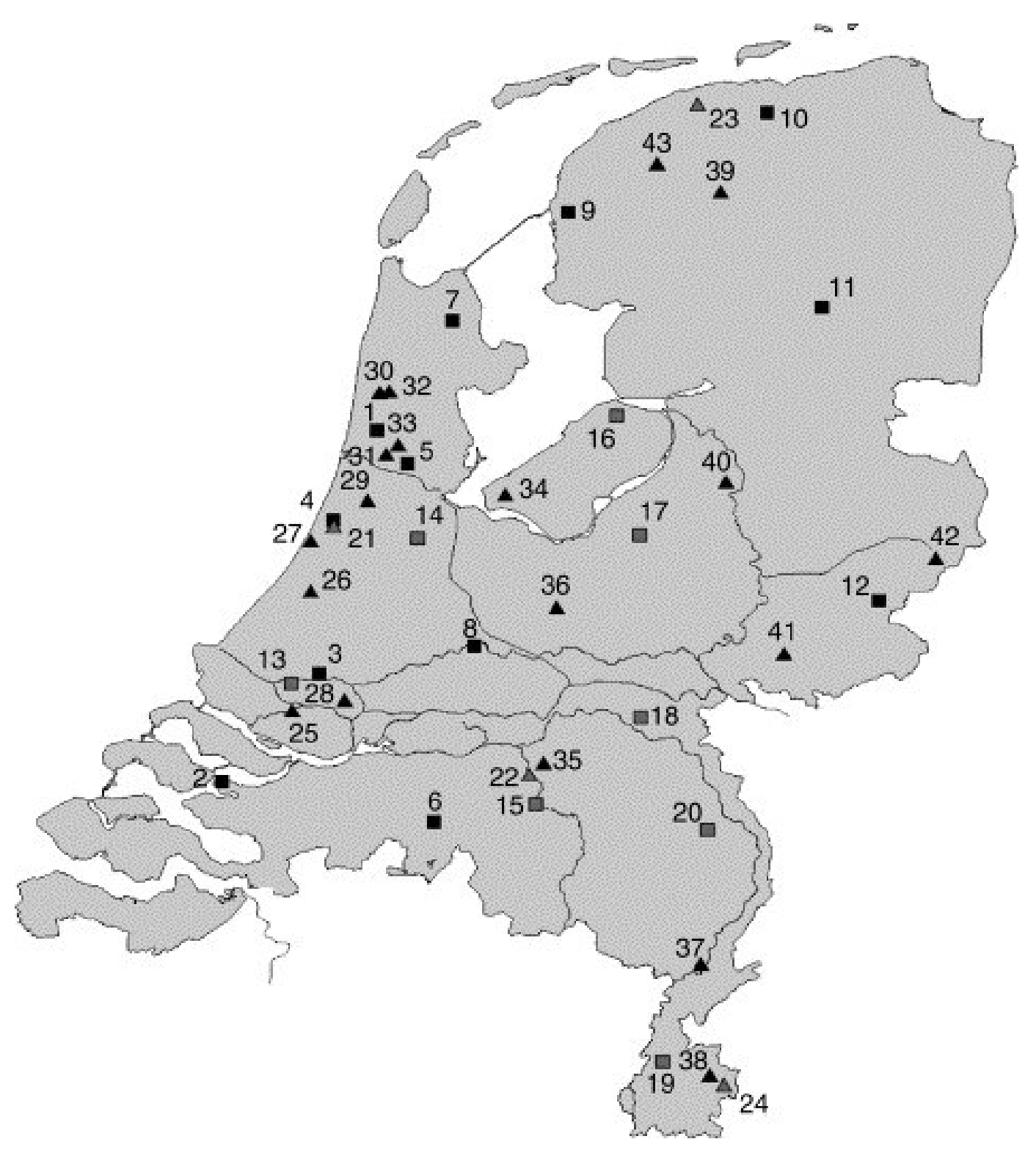

Fig. 2. Overview of sampling locations in the Netherlands for freshwater sediment only (gray triangles), freshwater sediment and fish (black triangles), soil only (black squares), and soil and vegetation (gray squares).

\subsection{Freshwater}

During spring, summer, and autumn 1999, 66 freshwater samples were taken from 23 sites for analysis of the dissolved concentration. Volumes ranged between $250 \mathrm{~mL}$ and $2 \mathrm{~L}$. From 11 of 23 locations, suspended material in the water column was sampled by means of a flow-through centrifuge. This yielded 32 samples ( 1 was lost) for the determination of the phthalate concentration in suspended material. Thus, 32 pairs of water and solid concentrations were obtained. The location and the numbering of the sites are given in Fig. 1. The LOD for dissolved DEHP was between 0.09 and $0.16 \mu \mathrm{g} \mathrm{L}^{-1}$ and that for DBP between 0.08 and $0.14 \mu \mathrm{g} \mathrm{L}^{-1}$. All suspended material samples contained DEHP above the LOD. The concentration of DBP in suspended solids could be 
determined in only a minor fraction of the 32 samples. The methodology of sampling and chemical analysis is given by Vethaak et al. (2002).

\subsection{Marine water and marine sediment}

During spring, summer, and autumn 1999, 12 marine samples were taken from four sites for analysis of the dissolved concentration. Suspended matter samples taken during this sampling were not analyzed for DBP, whereas DEHP was determined in 9 samples collected from three sites. LODs for DBP and DEHP were similar to the LODs for the freshwater compartment.

\subsection{Freshwater sediment}

In early 1999, 30 sediment samples were collected in the Netherlands (see Fig. 2 for the location and the numbering of the sites). The median organic carbon content was $4.6 \%$, ranging from less than $0.5 \%$ (clean pond in Oud Beijerland, site 28 in Fig. 2) to $14 \%$ in Wormerveer (site 31 in Fig. 2). The amount of particles smaller than $1 \mu \mathrm{m}$ varied from $1.7 \%$ (Noordwijk, site 27 in Fig. 2) to $26 \%$ in Alkmaar (site 29 in Fig. 2) and Assendelft (site 7 in Fig. 2), with a median equal to $9.4 \%$. After addition of the internal standard (d4-DEHP), approximately $10 \mathrm{~g}$ homogenized sample was extracted with $10 \mathrm{~mL}$ methanol in a $40-\mathrm{mL}$ vial for $30 \mathrm{~min}$ using sonication. After this first extraction, $10 \mathrm{~mL}$ cyclohexane was added and the extraction was repeated using sonication for $30 \mathrm{~min}$, followed by horizontal shaking for $30 \mathrm{~min}$. Finally, the vials were again placed in the ultrasonic bath for $30 \mathrm{~min}$. Then the sediment material was filtered off and the liquid phase was collected in another $40 \mathrm{~mL}$ vial. To the methanol/cyclohexane fraction, $20 \mathrm{~mL}$ water was added (to wash out the methanol from the cyclohexane phase) and an aliquot of the (upper) cyclohexane phase was transferred to an autosampler vial. The extracts were analyzed by GC-MS (Pauwels et al., 1999; Tienpont et al., 2000). Analyses were performed on a HP $6890 \mathrm{GC}$ in combination with a HP 5973 MSD. Separation was done on a $30 \mathrm{~m} \times 0.25 \mathrm{~mm}$ i.d. $\times 0.25 \mu \mathrm{m}$ HP-5MS column. Injection was done in splitless mode. The oven was programmed from $50^{\circ} \mathrm{C}(1 \mathrm{~min})$ to $320^{\circ} \mathrm{C}(5 \mathrm{~min})$ at $15^{\circ} \mathrm{C} \mathrm{min}{ }^{-1}$. Calibration was done by injection of standard solutions of phthalates in cyclohexane. Data are blank corrected and expressed in $\mu \mathrm{g} \mathrm{kg}^{-1}$ wet sample. Dry masses were determined on separate aliquots of the homogenized samples drying at $105^{\circ} \mathrm{C}$ by $16 \mathrm{~h}$. The LODs for DEHP and DBP in sediment were 15 and $25 \mu \mathrm{g} \mathrm{kg} \mathrm{dry} \mathrm{weight}{ }^{-1}$, respectively. Recovery of d4-DEHP was between $95 \%$ and $105 \%$.

\subsection{Fish in freshwater}

Local fishermen collected 25 samples of bream and roach at different locations in the Netherlands in 1999 (see Fig. 2 for the locations and the numbering of the sites). These fish species were selected since roach are exposed primarily via the water phase whereas bream are exposed via both the aqueous phase and the sediment. The lipid content of the fish varied between $0.1 \%$ and $5.1 \%$ (median $0.5 \% ; 90$ th percentile $2.2 \%$ ). Fish samples were stored at $-40{ }^{\circ} \mathrm{C}$. After thawing, heads and tails were removed. The remaining parts were homogenized using a household blender. Hot water, bidistilled water, acetone, and cyclohexane were used between samples to clean the blender. The homogenized samples were stored at $-18{ }^{\circ} \mathrm{C}$ until extraction. Approximately $25 \mathrm{~g}$ homogenized sample was extracted with $50 \mathrm{~mL}$ of a cyclohexane/acetone mixture (1/1) in a $250-\mathrm{mL}$ Erlenmeyer flask for 30 min using sonication, placed on a shaking machine for $30 \mathrm{~min}$, and again placed in the ultrasonic bath for $30 \mathrm{~min}$. After completion, the material was allowed to settle. The supernatant was filtered and centrifuged. An aliquot $(10 \mathrm{~mL})$ of the upper layer was isolated and evaporated to dryness under 
nitrogen (Turbovap, Zymark) in a preweighed vial. After evaporation the extract weight was determined (=fat content). Sample cleanup was performed by gel permeation chromatography and solid-phase extraction. The residue of the extraction was dissolved in $1 \mathrm{~mL}$ dichloromethane and $10 \mu \mathrm{L}$ of a $10-\mathrm{ng} / \mu \mathrm{L}$ internal standard solution (d4-DEHP) was added. Further cleanup and analysis were as described by Pauwels et al. (1999). The LOD was $100 \mathrm{ng} \mathrm{g}^{-1}$ fat of the fish for both phthalates.

\subsection{Soil and vegetation}

During the summer and autumn 1997 and spring and summer 1998, 34 soil samples and 47 vegetation samples were taken. Vegetation sampled included mixed grass samples and cabbage. Fig. 2 contains information on the sampling sites. The dry weight content of soil ranged from 63\% to $96 \%$ (median $83 \%$ ); dry matter of vegetation varied between $11 \%$ and $64 \%$ (median $18.6 \%$ ). Soil samples were treated as described above for sediment samples; vegetation samples were treated similar to fish samples. An additional cleanup using solidphase extraction on a Florisil cartridge was used to remove plant sterol material which was found to interfere with the analyses.

The LODs for DEHP and DBP in soil were 15 and $25 \mu \mathrm{gg}^{-1}$ dry weight, respectively. For vegetation, $50 \mu \mathrm{g} \mathrm{kg}^{-1}$ dry weight was reported as the LOQ of both phthalates. "Indicative" levels were reported in the range between 1 and $50 \mu \mathrm{kg}^{-1}$ dry weight.

\subsection{Air}

Air samples were taken at four sites in February 2000, August 2000, February 2001, and August 2001. One site (Pernis) is located in a highly industrialized area in the Netherlands, another site (Vianen) is located in a heavily populated area, and two other sites represent background locations. Sampling was performed on sorption tubes packed with $5 \%$ polydimethylsiloxane on Chromosorb. Sampling

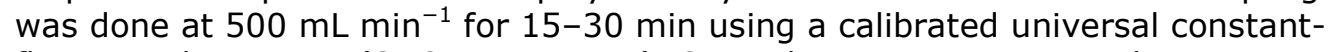
flow sampling pump (SKC, Dorset, UK). Special precautions were taken to avoid contamination. All sampling tubes were preconditioned and checked for blank levels of phthalates before sampling. The analyses were performed by thermal desorption-GC-MS. The thermal desorption tubes were desorbed at $300{ }^{\circ} \mathrm{C}$ for $10 \mathrm{~min}$. The desorbed compounds were analyzed by GC-MS in scan mode. The validation of the sampling method and analysis is described by Tienpont et al. (2000). Results were corrected for travel blank values. In general the travel blank levels corresponded to the analytical detection limits. The analytical LOD for single isomer phthalates was $15 \mathrm{pg}$. For a $15 \mathrm{~L}$ sample, this corresponded to $1 \mathrm{ng} \mathrm{m}^{-3}$. The method LOQ was set at $2 \times$ the analytical LOD or $2 \mathrm{ng} \mathrm{m}^{-3}$. The sampling locations are shown in Fig. 1.

\section{Treatment of measured data}

The general premise was that the concentrations of DEHP and DBP in all compartments show a log-normal distribution. It should be noted that this premise at first glance is contradictive of the basic assumptions in selection of the sampling sites, i.e., the dataset was supposed to include sample locations where low and high concentrations of contaminants (not necessarily phthalate esters) were expected. The DEHP and DBP concentrations above LOD were tested in a quantile-quantile ( $Q-Q$ ) plot to verify the log-normal distribution. A Q-Q plot is a graphical display used to compare a dataset (empirical) to a theoretical probability distribution (assumption). If both distributions are the same, the Q-Q 
plot is a straight line with an abscissa of zero $(y=x)$. If both distributions have different locations and shape parameters but both are samples from the same distributions, the $\mathrm{Q}-\mathrm{Q}$ plot is a straight line $(y=a x+b)$. The verification of the distribution of the DEHP and DBP concentrations above LOD showed, however, that the assumption of a log-normal distribution is not violated by the dataset collected. Cumulative probability plots; the geometric mean (GM), and the geometric standard deviation (GSD) were calculated from log-transformed concentrations.

Measured concentrations in sediment samples were expressed in units of $\mathrm{mg} \mathrm{kg}^{-1}$ dry weight. Substitute values for freshwater sediment samples with concentrations below the detection limit were calculated using the method described by Swaving and De Vries (2000). This value $\left(x_{i}\right)$ is calculated according to the following equation:

$$
x_{i}=\operatorname{LOD}\left[\left(\frac{\mathrm{LOD}}{\mathrm{x}_{0.9}}\right)^{\mathrm{frac}}\right] \text { with : frac } \leqslant 0.5 \text {, }
$$

in which the fraction of censored data, frac, is lower than or equal to $1 / 2$ and $x_{0.9}$ is the 90th percentile of the dataset. This method was used since it provides more reliable estimates than the common procedure of substituting values below the detection limit with a value equal to half of the detection limit. All concentrations (which were originally in units of $\mu \mathrm{g} \mathrm{kg}^{-1}$ wet sediment) were subsequently multiplied by the corresponding dry weight fractions to obtain a set of concentrations in units of $\mu \mathrm{g} \mathrm{kg}^{-1}$ dry sediment. The GM and GSD were calculated from this dataset.

Concentrations in 25 fish samples were determined in the lipid fraction of the fish, with a LOD of $100 \mathrm{ng} \mathrm{g}^{-1}$ for both phthalates. The results were converted into units of $\mu \mathrm{g} \mathrm{kg}^{-1}$ wet fish, with LOD equal to $1 \mu \mathrm{g} \mathrm{kg}^{-1}$ wet fish. Substitute values were again calculated according to Eq. (1). From this dataset GM and GSD were calculated. Note that in this case, distinct to for instance the freshwater samples, there is only one LOD. This is due to the fact that all samples were analyzed simultaneously.

\section{Results and discussion}

\subsection{Freshwater column}

In one freshwater sample the dissolved concentration of DEHP was $1000 \times$ higher than the average and therefore it was removed from the dataset. The LOD appeared not to be a single value but varied between 0.09 and $0.16 \mu \mathrm{g} \mathrm{L}^{-1}$. Because only a small minority of the measured phthalate concentrations (dissolved) in surface water were below this limit, for pragmatic reasons <LOD was replaced with LOD/2 instead of using the method of Swaving and De Vries (2000). In view of the limited number of data $\angle L O D$, we anticipate that this will not affect the outcome of the analysis.

Concentrations of DEHP and DBP measured as dissolved and sorbed to suspended matter are given in Table 2 and represented as empirical cumulative distribution functions (CDF plots) in Fig. 3. 
Table 2. DEHP and DBP concentrations $\left(\mu \mathrm{g} \mathrm{L}^{-1}\right)$ dissolved in freshwater (top) and comparison of DEHP concentrations in suspended solids $\left(C_{\mathrm{s}}\right.$ in $\left.\mu \mathrm{g} \mathrm{kg}^{-1}\right)$ and in water (bottom)

\begin{tabular}{|c|c|c|c|c|c|c|}
\hline \multirow[t]{2}{*}{ Location } & \multicolumn{2}{|c|}{ Spring } & \multicolumn{2}{|c|}{ Summer } & \multicolumn{2}{|c|}{ Autumn } \\
\hline & DEHP & DBP & DEHP & DBP & DEHP & DBP \\
\hline Biesbosch (Gat van de Kerksloot) & 0.18 & 0.16 & 0.22 & 0.27 & 0.28 & 0.19 \\
\hline Border Meuse (Roosteren) & & & 0.32 & 0.36 & 0.16 & 0.32 \\
\hline Brakel & 0.18 & 0.20 & Outlier $^{\mathrm{a}}$ & 1.88 & 0.84 & 0.58 \\
\hline Canal Apeldoorn & 0.27 & 0.15 & & & & \\
\hline De Dommel & 0.20 & 0.13 & & & & \\
\hline Ditch agriculture under glass & 0.45 & 0.80 & & & 0.05 & $0.09^{b}$ \\
\hline Haringvliet sluices & 0.21 & 0.16 & 1.13 & 0.92 & 0.36 & 0.33 \\
\hline Keizersveer & 0.40 & 0.11 & 0.31 & 0.16 & 0.10 & 0.07 \\
\hline Koudevaart (St Annaparochie) & 0.27 & 0.22 & 1.64 & 0.18 & 0.59 & 0.28 \\
\hline Lake Bergum & 0.25 & 0.19 & 2.35 & 0.28 & 1.10 & 0.15 \\
\hline Lake IJssel (Andijk) & 0.43 & 0.50 & 0.44 & 0.47 & 0.49 & 0.10 \\
\hline Lake IJssel (Vrouwenzand) & 0.24 & 1.07 & 0.69 & 0.20 & 1.45 & 0.63 \\
\hline Lateraal Canal Heel & & & 0.34 & $0.04^{b}$ & 0.20 & 0.11 \\
\hline Lekkerkerk & $0.05^{\mathrm{b}}$ & $0.04^{b}$ & 0.42 & 0.40 & 0.10 & 0.13 \\
\hline Meuse (Eijsden) & 0.28 & 0.31 & 0.62 & 1.33 & 0.36 & 0.10 \\
\hline Nieuw Lekkerland & $0.05^{b}$ & 0.14 & $0.08^{b}$ & 0.14 & 0.41 & 0.03 \\
\hline Nieuwe Waterweg (Maassluis) & 0.25 & 0.15 & 1.60 & 0.30 & 0.22 & 1.32 \\
\hline Nordsea Canal (Amsterdam) & 0.37 & 0.14 & 0.25 & 0.39 & 0.27 & 0.62 \\
\hline Nordsea Canal (IJmuiden) & 0.56 & 0.08 & 0.57 & 0.73 & 0.48 & 1.02 \\
\hline Ouddorp & & & 0.32 & 0.18 & 0.13 & 0.04 \\
\hline Rhine (Lobith) & 0.58 & $0.04^{b}$ & 0.92 & 0.29 & 0.54 & 0.20 \\
\hline Scheldt (Schaar van Oudendoel) & 0.30 & $0.04^{b}$ & 0.17 & 0.42 & $0.07^{b}$ & 0.25 \\
\hline Surface water (Canal) & & & & & 0.25 & $0.09^{b}$ \\
\hline Surface water De Dommel & & & & & 4.96 & 0.67 \\
\hline Twente Canal & 0.41 & 0.24 & 0.36 & 0.23 & 0.90 & 0.41 \\
\hline WRK Nieuwegein & 0.24 & $0.04^{b}$ & 0.27 & 0.21 & 0.25 & 0.15 \\
\hline Average & 0.28 & 0.23 & 0.65 & 0.45 & 0.61 & 0.33 \\
\hline \multirow[t]{2}{*}{ Median } & 0.27 & 0.15 & 0.39 & 0.30 & 0.32 & 0.20 \\
\hline & & Spring & Summer & Autumn & & \\
\hline \multirow[t]{2}{*}{ De Dommel } & $C_{\mathrm{s}}$ & 8481 & n.d. & 11,809 & & \\
\hline & $C_{\mathrm{w}}$ & 0.20 & n.d. & 4.96 & & \\
\hline \multirow[t]{2}{*}{ Haringvliet sluices } & $C_{\mathrm{s}}$ & 3734 & 10,768 & 2944 & & \\
\hline & $C_{\mathrm{w}}$ & 0.21 & 1.13 & 0.36 & & \\
\hline \multirow[t]{2}{*}{ Koudevaart (St Annaparochie) } & $C_{\mathrm{s}}$ & 1039 & 1404 & 2662 & & \\
\hline & $C_{\mathrm{w}}$ & 0.27 & 1.64 & 0.59 & & \\
\hline \multirow[t]{2}{*}{ Lake Bergum } & $C_{\mathrm{s}}$ & 876 & 697 & 970 & & \\
\hline & $C_{\mathrm{w}}$ & 0.25 & 2.35 & 1.1 & & \\
\hline \multirow[t]{2}{*}{ Lake IJssel (Vrouwenzand) } & $C_{\mathrm{s}}$ & 792 & 2300 & 8900 & & \\
\hline & $C_{\mathrm{w}}$ & 0.24 & 0.69 & 1.45 & & \\
\hline \multirow[t]{2}{*}{ Meuse (Eijsden) } & $C_{\mathrm{s}}$ & 9858 & 14,598 & 10,849 & & \\
\hline & $C_{\mathrm{w}}$ & 0.28 & 0.62 & 0.36 & & \\
\hline \multirow[t]{2}{*}{ Nieuwe Waterweg (Maassluis) } & $C_{\mathrm{s}}$ & 2906 & 3215 & 5601 & & \\
\hline & $C_{\mathrm{w}}$ & 0.25 & 1.6 & 0.22 & & \\
\hline \multirow[t]{2}{*}{ Nordsea Canal (Amsterdam) } & $C_{\mathrm{s}}$ & 10,483 & 3821 & 7854 & & \\
\hline & $C_{w}$ & 0.37 & 0.25 & 0.27 & & \\
\hline \multirow[t]{2}{*}{ Nordsea Canal (IJmuiden) } & $C_{\mathrm{s}}$ & 5843 & 3625 & 19,258 & & \\
\hline & $C_{w}$ & 0.56 & 0.57 & 0.48 & & \\
\hline \multirow[t]{2}{*}{ Rhine (Lobith) } & $C_{\mathrm{s}}$ & 2993 & 2149 & 4802 & & \\
\hline & $C_{\mathrm{w}}$ & 0.58 & 0.92 & 0.54 & & \\
\hline \multirow[t]{2}{*}{ Scheldt (Schaar van Oudendoel) } & $C_{\mathrm{s}}$ & 11,400 & 6808 & 13,178 & & \\
\hline & $C_{\mathrm{w}}$ & 0.30 & 0.17 & 0.065 & & \\
\hline
\end{tabular}

In case no value is given, the site was not sampled during the specific season indicated. ${ }^{\mathrm{a}}$ Reported value: $200.4 \mu \mathrm{g} \mathrm{L}{ }^{-1} .{ }^{\mathrm{b}} 1 / 2$ detection limit. 

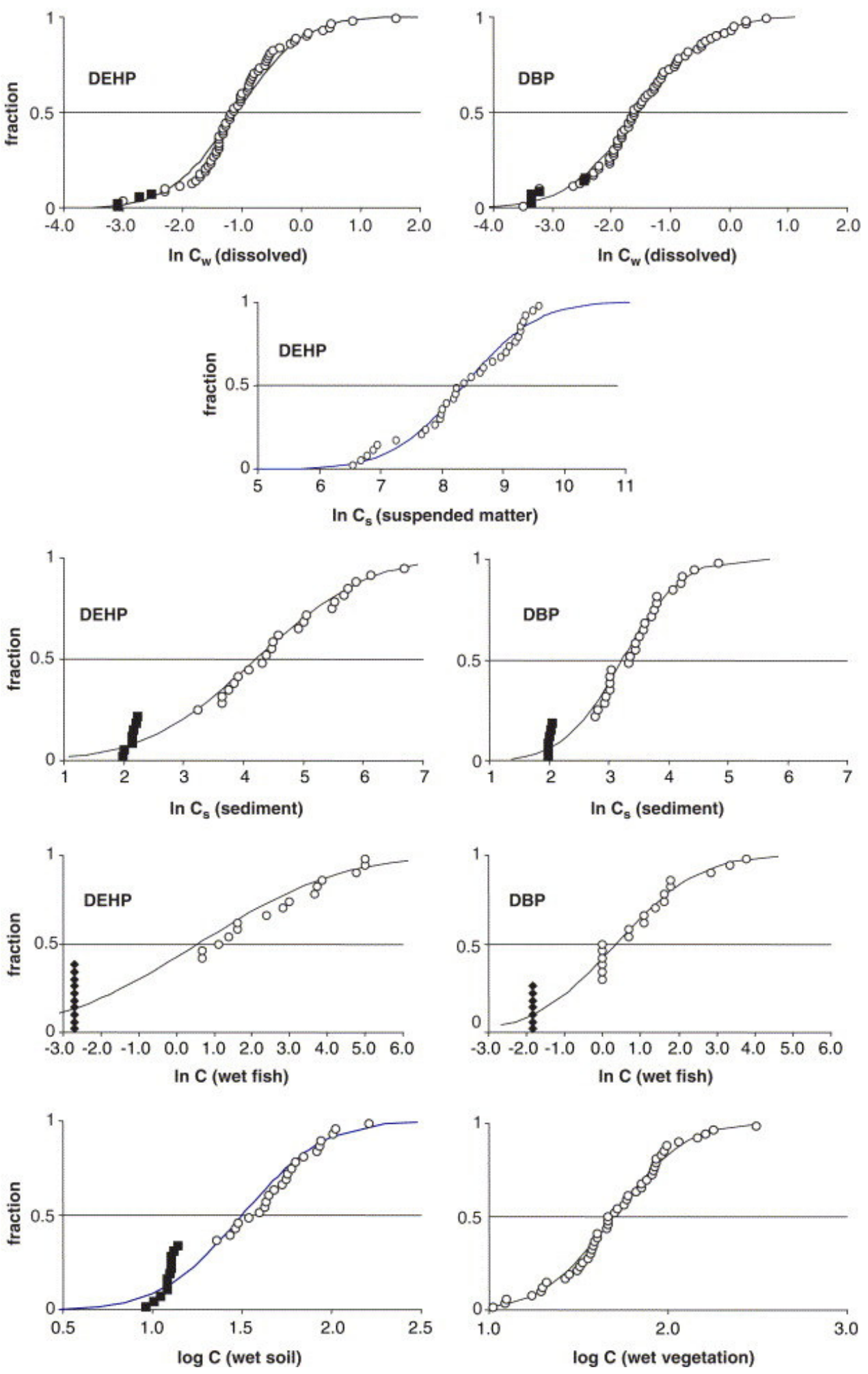

Fig. 3. CDF plots and Hazen plotting positions of concentrations dissolved in freshwater $\left(C_{\mathrm{w}}\right.$ in $\left.\mu \mathrm{g} \mathrm{L}^{-1}\right)$, (dry) suspended solids $\left(C_{\mathrm{s}}\right.$ in $\left.\mu \mathrm{g} \mathrm{kg}^{-1}\right)$, freshwater sediment ( $C_{\mathrm{s}}$ in $\mu \mathrm{g} \mathrm{kg}^{-1}$ wet weight), fish ( $\mu \mathrm{g} \mathrm{kg}{ }^{-1}$ wet weight), and soil and vegetation ( $\mu \mathrm{g} \mathrm{kg}^{-1} \mathrm{dry}$ weight). Black squares are values substituted for censored data. 
From the data presented in Table 2 it may be concluded that the highest levels of DEHP and DBP are found in summer and autumn, with DEHP usually present in the highest concentrations. These results are in good agreement with earlier measurements in the Netherlands in 1997 (Jonker et al., 1998). Vethaak et al. (2002) reported that, in general, levels of phthalates in surface waters are low, although phthalates are found in all samples. DEHP is the most abundant isomer; levels of dimethylphthalate, dipropylphthalate, dicyclohexylphthalate, and dioctylphthalate are always very low (i.e., in the study of Vethaak et al. (2002): less than $0.2 \mu \mathrm{g} \mathrm{L}^{-1}$ ). For the other isomers (such as diethylphthalate, dibutylphthalate, and buytlbenzylphthalate) a broader range in concentrations is found.

Analyzing DBP in suspended material appeared problematic. The LOD was different in different seasons, but within one season the LODs were the same for each sample, and the LOD depended on the measurement series only. In 9

samples the result of the determination was reported as "below $2008 \mu \mathrm{gg}^{-1}$ " (all summer data), in 1 sample this limit was 665 (spring), in 4 samples 451 (also spring); in 1 sample 52 (autumn), and in 4 samples 51 (also autumn) $\mu \mathrm{kg}^{-1}$. Only in 12 samples was determination possible, resulting in lowest and highest values of 34 and $4129 \mathrm{mg} \mathrm{kg}^{-1}$, respectively. The median of 12 concentrations was $88 \mathrm{mg} \mathrm{kg}^{-1}$. In view of the limited amount of reliable data, no CDF plot is given for DBP in suspended solids in Fig. 3.

\subsection{Marine water and marine sediment}

DEHP concentrations in marine sediments deviated significantly from the detection limit in 4 of the 9 samples analyzed. Detectable concentrations of DEHP were found in just 3 of the 12 water samples, whereas in all cases DBP concentrations in marine sediments were below the detection limit. Although the amount of data is limited, concentrations of phthalates in marine waters are of the same order of magnitude as those in freshwaters. The data do not allow distinguishing clear trends among seasons, and the amount of data is considered insufficient for further statistical treatment of the data collected in the marine environment.

\subsection{Freshwater sediment}

In sediment samples, $23 \%$ (DEHP) and 20\% (DBP) of the samples were below LOD (Fig. 3). The dataset including substituted values fit the CDF curve reasonably well. The distribution of DEHP concentrations in sediment is wider than that of DBP concentrations.

\subsection{Fish}

In 10 of 25 samples the concentration of DEHP was below LOD, whereas in 7 samples DBP levels were below LOD. The probability plots in Fig. 3 show a roughly $10 \times$ wider distribution of DEHP concentrations compared to DBP levels. The GMs were 1.8 and $1.0 \mu \mathrm{g} \mathrm{kg}^{-1}$ wet fish for DEHP and DBP, respectively (see also Table 3). 
Table 3.

Average of duplicate concentrations in air $\left(\mathrm{ng} \mathrm{m}^{-3}\right)$

\begin{tabular}{|l|l|l|}
\hline Location (No in Fig. 1) & DEHP & DBP \\
\hline Gilze-Rijen (25) & 25 & 19 \\
\hline & 57 & 25 \\
\hline. & $0.73^{\mathrm{a}}$ & 5 \\
\hline Pernis (24) & 31 & 9 \\
\hline & 333 & 70 \\
\hline & $0.73^{\mathrm{a}}$ & 4 \\
\hline & 48 & 7 \\
\hline Speulderveld (27) & 8 & 11 \\
\hline & 9 & 31 \\
\hline & $0.73^{\mathrm{a}}$ & 7 \\
\hline & 20 & 2 \\
\hline Vianen (23) & 52 & 9 \\
\hline & 72 & 17 \\
\hline & $0.73^{\mathrm{a}}$ & 5 \\
\hline & & \\
\hline
\end{tabular}

Each value corresponds to one sampling date. The numbers between paranthesis indicate the total number of samples taken.

a Substituted for $<2$.

\subsection{Soil and vegetation}

In 12 of 34 soil samples the concentration of DEHP was below LOD. Two vegetation samples were not analyzed for DEHP and therefore not further considered part of the dataset; the remaining 45 samples appeared above LOD (Fig. 3). The GM values were 31.8 and $41.8 \mu \mathrm{g} \mathrm{kg}^{-1}$ (wet) for soil and vegetation, respectively. Both distributions display a comparable width (soil: GSD=2.3; vegetation: $G S D=2.9$ ). Most soil and vegetation samples contained DBP concentrations below the LOD. The median DBP concentration in soil, $6.0 \mu \mathrm{g} \mathrm{kg}^{-1}$ (wet), was estimated through extrapolation according to Swaving and De Vries (2000). It appeared relatively close to the GM (6.7) calculated from the whole dataset, including substituted values below LOD which range from 3.1 to $4.5 \mu \mathrm{gg}^{-1}$ (wet). The 95th percentile $\left(29.1 \mu \mathrm{g} \mathrm{kg}^{-1}\right)$ relative to the median was used to quantify the width of the distribution.

The median concentration in vegetation was calculated in a similar way. The difference with respect to the GM of all data (included substituted values), $0.54 \mu \mathrm{g} \mathrm{kg}^{-1}$ (wet), was greater than that for soil. The accuracy, however, is poor due to the fact that most of the data were indicative values. This may have caused the rather high dispersion: the 95th percentile exceeds the median by a factor of 14 .

\subsection{Air}

In all samples duplicate concentrations of DBP did not deviate more than $30 \%$ from the mean and were above the detection limit of $2 \mathrm{ng} \mathrm{m}^{-3}$ (Table 3). From a $\mathrm{Q}-\mathrm{Q}$ plot the conclusion is justified that a log-normal distribution is most likely (Fig. 4) since the data shown in Fig. 4 can well be represented by a straight line. The CDF is given in Fig. 4 also. In this figure the measured concentrations are represented by Hazen plotting positions (Cunnane, 1978). These are midpoints halfway empirical CDF jumps. 


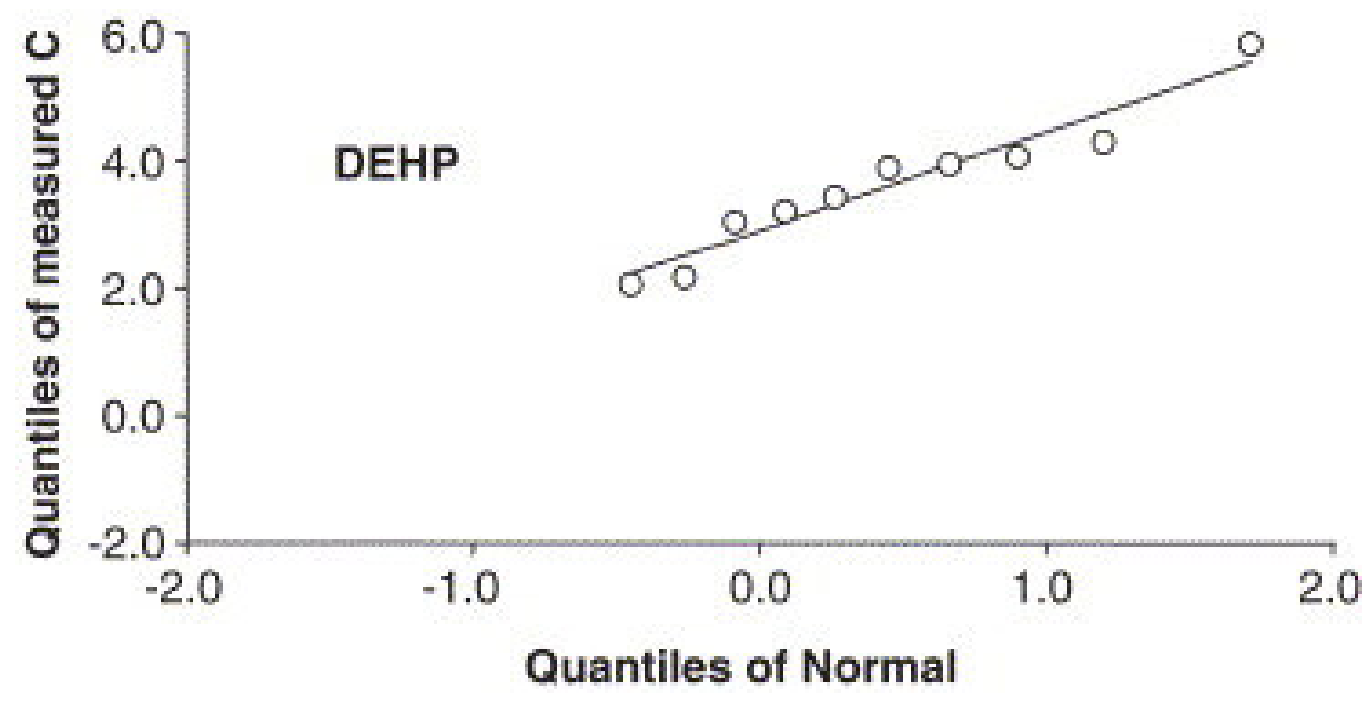

Fig. 4. Q-Q plot of log-transformed DEHP concentrations in air samples (top) and cumulative probability plots and Hazen plotting positions of phthalate concentrations in air bottom. The solid circles in the left side plot at bottom represent substituted data $\left(0.73 \mathrm{ng} \mathrm{m}^{-3}\right)$ for determinations of DEHP below LOD.

Interpretation of the DEHP dataset was more difficult. The concentrations show a wider dispersion compared to DBP. Nevertheless, the duplicates met the quality criteria since they differ less than $20 \%$ from the mean, with the exception of one sample $(33 \%)$. There were no reasons to suspect the data of analytical artifacts or to consider them outliers. Concentrations below the sample blank $\left(2 \mathrm{ng} \mathrm{m}^{-3}\right)$ were substituted by $0.73 \mathrm{ng} \mathrm{m}^{-3}$, according to the method of Swaving and De Vries (2000).

\subsection{Significance of the observed concentrations}

Table 4 contains median and 95th percentile concentrations of assumed lognormally distributed environmental concentrations of DEHP and DBP in six environmental compartments in the Netherlands.

Table 4.

Median and 95th percentile values of assumed log-normally distributed environmental concentrations of DEHP and DBP in the Netherlands

\begin{tabular}{|l|l|l|l|l|l|}
\hline Compartment & Units & DEHP & DBP & \\
\hline & & Median & 95th Perc. & Median & 95th Perc. \\
\hline Outdoor air & & 11.9 & 163.4 & 10.3 & 44.7 \\
\hline Freshwater dissolved & $\mathrm{ng} \mathrm{m}^{-3}$ & 0.33 & 1.56 & 0.21 & 1.06 \\
\hline Freshwater susp. Sediment & $\mu \mathrm{g} \mathrm{kg}^{-1}(\mathrm{dry})$ & 4311 & 13800 & $88^{\mathrm{a}}$ & $372^{\mathrm{a}}$ \\
\hline Sediment & $\mu \mathrm{kg}^{-1}$ (wet) & 67.4 & 649 & 25.3 & 77.5 \\
\hline Fish & $\mu \mathrm{kg}^{-1}$ (wet) & 1.7 & 141 & 1 & 26 \\
\hline
\end{tabular}

${ }^{a}$ Result of 12 determinations of 32 samples.

Fugacity: Table 4 does not disclose whether there is equilibrium among the compartments. The fugacity is a thermodynamic quantity related to the chemical activity (or concentration) in some compartment or phase that characterizes the escaping capacity of the chemical from one phase or compartment to another 
(Mackay et al., 1985). All fugacities of a compound are equal at equilibrium. The fugacity in water is estimated from the dissolved concentration in water, the water solubility, and the vapor pressure. Water solubility and vapor pressure are temperature dependent. In soil and sediment the partition coefficients and density are needed also (Table 5).

Table 5.

Expressions used for calculating fugacity (all concentrations $\left(C_{g, w, s, s e d .}\right)$ and water solubility $(\mathrm{S})$ in mol m${ }^{-3} ; K_{\mathrm{p}}$ in $\mathrm{L} \mathrm{kg}^{-1} ; \rho$ in $\mathrm{kg} \mathrm{L}^{-1}$; vapor pressure $\left(V_{p}\right)$ in $\left.\mathrm{Pa}\right)$ and temperature-dependent vapor pressure and water solubility of DEHP and DBP used to convert water concentrations into fugacities

\begin{tabular}{|c|c|c|c|c|}
\hline Fugacity (Pa) & Expression & \multicolumn{3}{|c|}{ Symbols, units } \\
\hline Air & $C_{g} R T$ & \multicolumn{3}{|c|}{$\begin{array}{l}C_{\mathrm{g}} \text { (air conc. not associated), } R \text { (universal gas constant, } \\
\left.\text { Pa } \mathrm{m}^{3} \mathrm{~mol}^{-1} \mathrm{~K}^{-1}\right), T \text { (temperature, } \mathrm{K} \text { ) }\end{array}$} \\
\hline f Water & $C_{\mathrm{w}} V_{p} S^{-1}$ & \multicolumn{3}{|c|}{$C_{\mathrm{w}}($ dissolved conc. $), V_{p}(\mathrm{~Pa}), \mathrm{S}$ (water solubility) } \\
\hline f Soil & $C_{\mathrm{s}} V_{p} S^{-1} K_{\mathrm{p}, \text { soil }}^{-1} \rho_{\text {soil }}^{-1}$ & \multicolumn{3}{|c|}{$\begin{array}{l}C_{s} \text { (conc. in soil), } K_{p, \text { soil }} \text { (partition coefficient), } \rho_{\text {soil }} \text { (density } \\
\text { soil) }\end{array}$} \\
\hline f Sediment & $C_{\text {sed }} V_{p} S^{-1} K_{p, \text { sed }}^{-1} \rho_{\text {sed }}^{-1}$ & \multicolumn{3}{|c|}{$\begin{array}{l}C_{\text {sed }} \text { (conc. in sed.), } K_{p, \text { sed }} \text { (partition coefficient), } \rho_{\text {sed }} \text { (density } \\
\text { sed.) }\end{array}$} \\
\hline \multirow[t]{2}{*}{$\begin{array}{l}\text { Temperature } \\
\left({ }^{\circ} \mathrm{C}\right)\end{array}$} & \multicolumn{2}{|l|}{ DEHP } & \multicolumn{2}{|l|}{ DBP } \\
\hline & $V_{p}(\mathrm{~Pa})$ & $S\left(\mathrm{~mol} \mathrm{~m}^{-3}\right)$ & $V_{p}(\mathrm{~Pa})$ & $S\left(\mathrm{~mol} \mathrm{~m}^{-3}\right)$ \\
\hline 8 (spring) & $3.9 \times 10^{-6}$ & $6.0 \times 10^{-6}$ & $1.4 \times 10^{-3}$ & $3.4 \times 10^{-2}$ \\
\hline 17 (summer) & $7.6 \times 10^{-6}$ & $6.9 \times 10^{-6}$ & $2.1 \times 10^{-3}$ & $3.6 \times 10^{-2}$ \\
\hline 12 (autumn) & $5.4 \times 10^{-6}$ & $6.4 \times 10^{-6}$ & $1.1 \times 10^{-3}$ & $3.2 \times 10^{-2}$ \\
\hline
\end{tabular}

As an approximation, the water samples during spring, summer, and autumn were assumed to be taken at 8,17 , and $12{ }^{\circ} \mathrm{C}$, respectively (Rijkswaterstaat, 2005). These values are the long-year average values that are typical for the Netherlands. The water solubility and the vapor pressure were adjusted accordingly (Table 5). Air temperature was recorded during sampling and the measured values were used for calculating fugacity.

Distributions of measured concentrations were converted into distributions of fugacities, and these are plotted in Fig. 5. Note that fugacities in air (DEHP/DBP) and in vegetation and soil (DEHP) are included in this figure. Examination of the plots given in Fig. 5 raises several important issues.

- The fugacity of DEHP in water is more than two orders of magnitude higher than that in air; apparently there is no equilibrium between air and water nor between sediment and water (difference more than a factor of 30 ).

- The highest fugacity of DEHP in water may indicate that water is the compartment to which emission is directly, whereas degradation in water is relatively slow (pseudo-first-order rate constant: 0.03 day $^{-1}$; according to Staples et al. (1997)). This implicates diffusive transport from water to air (where degradation is fast) and to other compartments.

- Sediment seems to be a sink for DEHP in the aquatic environment. Transport of DEHP from water to sediment is merely due to settling of suspended material carrying the adsorbed chemical with it. The concentration in sediment is governed by the rates of supply of DEHP (through sedimentation) and degradation. These fugacity plots could be an indication that biodegradation in sediment might be faster than the rate constant given by Staples et al. (1997) $\left(0.003\right.$ day $\left.^{-1}\right)$. 
- Biotransformation of DEHP in fish seems to be relatively fast: the median fugacity is several orders of magnitude lower than that in water.

- Concentrations of DBP in air, water, and sediment are close to equilibrium: the fugacities of DBP in these compartments do not span more than a factor of five.

- For DBP the median fugacity in sediment is only a factor of two lower than that in water, indicating that equilibrium is approximated. According to Parkerton and Konkel (2001) and two EU Risk Assessment Report, 2001a and EU Risk

Assessment Report, 2001b, water would be the primary compartment for DBP to which DBP is emitted. However, the fugacity plot of DBP suggests that air is the primary compartment. A relatively high emission may be balanced by rapid biodegradation in the water compartment $\left(t_{1 / 2}=7\right.$ days).
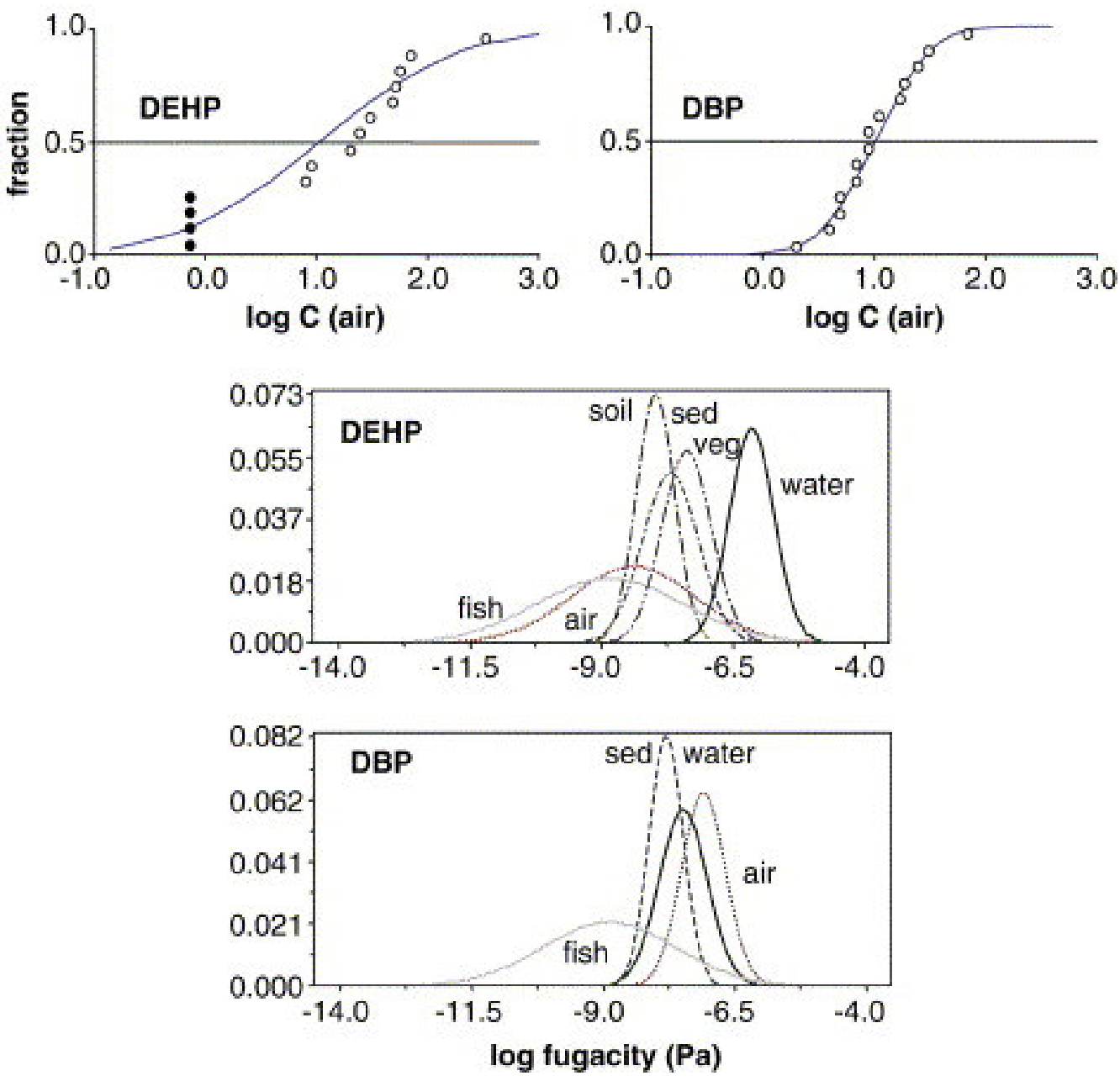

Fig. 5. Fugacity distributions calculated from measured concentrations in environmental compartments. The $y$-axis indicates the probability of detection of either DEHP or DBP in the compartment indicated at the specified value of logfugacity.

It should be noted that this discussion of fugacity is based on the assumption that the whole Dutch environment can be considered a homogeneous system. This assumption may not be true for anthropogenic contaminants such as phthalates, although phthalates are ubiquitous in the environment and have been present for 
many decades. In addition it should be noted that water, air, and sediment samples were collected at different times and at different sites. Variances in time and sampling location too may, especially for water, affect the calculated fugacities. As sampling covered various seasons and as the geographical distribution of the sampling sites is representative for the Netherlands, it is unlikely that the deviations of orders of magnitude as found in this study are due to variances in sampling time and location.

Suspended solids-water partitioning: The lack of overlap of the calculated fugacity distributions (Fig. 5) indicates that there is no equilibrium between suspended solids and water for DEHP. Nevertheless, a set of $32 K_{\mathrm{p} \text {,susp. values was }}$ calculated for DEHP from the 32 pairs of concentrations given in Table 2 . The 5 th, 50th and 95th percentiles are 870,9211 , and $41,149 \mathrm{mg} \mathrm{kg}^{-1}$, respectively. The mean and median of log-transformed data coincide at 3.95, suggesting that the distribution of field $K_{\mathrm{p}, \text { susp }}$ is log-normal.

The ratio of $C_{s}$ and the corresponding dissolved concentration yielded 12 values of $K_{\mathrm{p}, \text { susp }}$ for DBP, which appear log-normally distributed (the median and mean of log-transformed data converge at 2.8).

Comparison of data reported to values found in other countries: Various databases containing data on phthalate isomers in various fresh surface waters in Western Europe, the USA, and Japan are available (see for instance Environment Agency Japan, 1995; Mabey, 1982). In general a clear distinction between phthalate levels found in pristine freshwaters and those in freshwaters impacted by anthropogenic influences needs to be made. DEHP levels in pristine waters are generally found to be around $0.01 \mu \mathrm{g} \mathrm{L}^{-1}$, whereas the ranges, median, and average values, of DEHP levels typically found in impacted freshwaters, correspond well with the ranges, median, and average values reported here. The same applies to DBP and to phthalate levels found in sediments. The majority of the studies cover a wide range of surface waters, sampled over prolonged periods of time, with the majority of studies being carried out during 1990-2002 (Fürtmann, 1993; Vethaak et al., 2002; Mabey, 1982; Environment Agency Japan, 1995).

\section{Conclusion}

From the results presented here it may be concluded that the most abundant phthalates DBP and DEHP can be found at low levels in most environmental compartments. Dependent on the season and the environmental compartment taken into account, often concentrations are found at or below the levels of detection. Whereas concentrations of DBP and DEHP in marine water and sediment are in most cases below the LOD of between 0.08 and $0.16 \mu \mathrm{g} \mathrm{L}^{-1}$, significantly higher levels of dissolved DBP and DEHP were present in freshwater samples. Median levels showed a log-normal distribution among the sites sampled, whereas statistical analysis as reported by Vethaak et al. (2002) showed that sampling period (sampling season) is not a relevant determinant parameter. Similar results were obtained for the freshwater sediment compartment, with DEHP levels exceeding concentrations of DBP. DPB levels in fish were often below the LOD and mean values of 1.8 and $1.9 \mu \mathrm{g} \mathrm{kg}^{-1}$ wet fish were found for DEHP and DBP, respectively. In all compartments sampled, no clear distinction in phthalate levels was found at sites presumed to represent noncontaminated areas and sites expected to be impacted by potential sources of contamination. 
Fugacity calculations revealed that especially for DEHP there is no equilibrium of phthalate isomers among the environmental compartments. From these calculations it can be deduced that water is the compartment to which DEHP emission is directed, whereas degradation in water is relatively slow. Sediments provide a sink for DEHP and there is net transport to air. Although it has been suggested that water is the primary compartment for DBP also, fugacity plots suggest that air is the compartment to which emission is directed dominantly. Finally, the data reported here are shown to be in line with values found in other Western European countries. As a note of caution it should be realized that, because of more or less continuing shifts in production patterns (and hence of use patterns) of the various phthalate isomers and mixtures of isomers, concentrations of phthalates not included in the monitoring campaign reported here may at some time intervals exceed the values reported here for DBP and DEHP. Currently, for instance, a clear shift is observed toward increased production of mixtures of phthalates based on technical mixtures of isomer precursors obtained by means of distillation.

\section{Acknowledgments}

Tom Parkerton (Exxon Mobil Biomedical Sciences, Inc., New Jersey, USA) and Frank David (Research Institute for Chromatography, Kortrijk, Belgium) are gratefully acknowledged for their valuable contribution in setting up and carrying out the sampling campaigns. Marga Schrap (RIZA), Dick Vethaak (RIKZ) and Pim de Voogt are acknowledged for critically reading earlier versions of the manuscript. 


\section{References}

Cunnane, 1978 C. Cunnane, Unbiased plotting positions-A review., J. Hydrology 37 (1978), pp. 205222.

ECETOC, 1988 ECETOC, 1988. Concentrations of industrial chemicals measured in the environment: the influence of physico-chemical properties, tonnage and use patterns. ECETOC Tech. Rep. No. 29.

Environment Agency Japan, 1995 Environment Agency Japan, Chemicals in the environment. Report on environmental survey and wildlife monitoring of chemicals, Environmental Health and Safety Division. Tokyo, Japan (1995).

EU Risk Assessment Report, 2001a EU Risk Assessment Report on DEHP, draft of 2001a. EC Regulation 793/93 (Existing Chemicals).

EU Risk Assessment Report, 2001b EU Risk Assessment Report on DBP, draft of 2001b. EC Regulation 793/93 (Existing Chemicals).

Fürtmann, 1993 Fürtmann, K., 1993. phthalate in der aquatischen umwelt. Landesamt für Wasser und Abfall Nordrein-Westfalen, Auf dem Draap 25, 40221 Düsseldorf (Germany).

Jonker et al., 1998 Jonker, N., Breebaart, L., Van der Velde, E.G., Ritsema, R., Hogendoorn, E.A., Versteegh, J.F.M., 1998. Resultaten meetprogramma drinkwater 1997. Dutch National Institute of Public Health and the Environment, Bilthoven, the Netherlands, Report No. 703713007 (In Dutch).

Lyman et al., 1990 W. Lyman, W. Reehl and D. Rosenblatt, Handbook of Chemical Property Estimation Methods: Environmental Behavior of Organic Compounds, American Chemical Society, Washington, DC (1990).

Mabey, 1982 Mabey, W.R., 1982. EPA Report No.440/4-81-014 by SRI International, Menlo Park, CA, USA.

Mackay et al., 1985 D. Mackay, S. Paterson, B. Chueng and W.B. Neely, Chemosphere 14 (1985), pp. 335-374.

Parkerton and Konkel, 2001 T. Parkerton and W. Konkel, Evaluation of the production, consumption, end use and potential emissions of phthalate esters. Prepared for the American Chemistry Council (Draft of November 2001), Exxon Mobil Biomedical Sciences Inc. (EMBSI), East Millstone, NJ, USA (2001).

Pauwels et al., 1999 A. Pauwels, F. David, P. Schepens and P. Sandra, Int. J. Environ. Anal. Chem. 73 (1999), p. 171.

Rijkswaterstaat, 2005 Rijkswaterstaat, 2005. www.waterbase.nl.

Staples et al., 1997 C.A. Staples, D.R. Peterson, T.F. Parkerton and W.J. Adams, Chemosphere 35 (1997), pp. 667-749.

Swaving and Vries, 2000 Swaving, M., De Vries, L., 2000. Projectrapport nr: E1680-01 Omgaan met waarden onder de detectiegrens. RIKZ/RIZA, the Netherlands (in Dutch).

Tienpont et al., 2000 B. Tienpont, F. David, P. Sandra and F. Vanwalleghem, J. Microcolumn Sep. 12 (2000), pp. 194-203.

Vethaak et al., 2002 Vethaak, A.D., Rijs, G.B.J., Schrap, S.M., Ruiter, H., Gerritsen, A., Lahr, J., 2002. Estrogens and xeno-estrogens in the aquatic environment of The Netherlands: occurrence, potency and biological effects. RIZA/RIKZ report no. 2002.001. ISBN 9036954010. Lelystad/The Hague (The Netherlands). 